\section{Morphological analysis and quantitative evaluation of myopic maculopathy by three- dimensional magnetic resonance imaging}

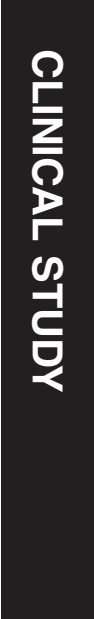

${ }^{1}$ State Key Laboratory of Ophthalmology, Zhongshan Ophthalmic Center, Sun Yat-sen University, Guangzhou, China

${ }^{2}$ The first affiliate hospital of Sun Yat-sen University, Guangzhou, China

Correspondence:

L Lu or Y Li, Zhongshan Ophthalmic Center, Sun Yat-sen University, Yuexiu District, Guangzhou, Guangdong, China Tel: +8620873300 00; Fax: +86 2087333271 . E-mail: drlulin@126.com or liyhao@mail.sysu.edu.cn

Received: 1 June 2017 Accepted in revised form: 23 September 2017 Published online: 9 February 2018

\author{
Abstract \\ Purpose To study the characteristic \\ morphology and quantitatively evaluate the \\ eye shape in different types of myopic \\ maculopathy. \\ Methods A total of 103 eyes from 65 patients \\ with high myopic maculopathy were \\ examined by spectral-domain optical \\ coherence tomography (SD-OCT) and three- \\ dimensional magnetic resonance imaging (3D \\ MRI). The participants were classified into \\ two groups, namely myopic traction \\ maculopathy (MTM) eyes and non-MTM \\ eyes, with SD-OCT imaging. Volume \\ renderings and morphology analysis of the \\ 3D MRI of the eyeball were obtained. \\ Quantitative analysis was achieved in the \\ calculation of vitreous volume and the three- \\ dimensional diameters of the eyeball in three \\ cardinal axes. The eye shape distribution and \\ the diameters of the eyeball were compared \\ between the two groups. Eye shape \\ distribution, vitreous volume, and eyeball \\ diameter were compared between MTM and \\ non-MTM eyes. \\ Results The MTM and non-MTM groups \\ had a total of 68 and 35 eyes, respectively. \\ A significant difference was found in the eye \\ shape distribution $(P<0.0001)$ between MTM \\ and non-MTM eyes. Most of the MTM eyes \\ had undergone a non-uniform expansion of \\ the eyeball, whereas the non-MTM eyes had \\ expanded uniformly. There was no significant \\ difference $(P>0.05)$ in either vitreous \\ volume or other diameters between the two \\ groups. \\ Conclusions Non-uniform globe expansion \\ and staphyloma formation might play an \\ important role in the pathogenesis of MTM. \\ Eye (2018) 32, 782-787; doi:10.1038/eye.2017.263; \\ published online 9 February 2018
}

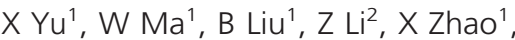
S Tanumiharjo ${ }^{1}, X$ Chen $^{1}, C \mathrm{Lyu}^{1}, \mathrm{H} \mathrm{Ao}^{1}, \mathrm{~S} \mathrm{Li}^{1}$, $Y \mathrm{Li}^{1}$ and $\mathrm{LLu}{ }^{1}$

\section{Introduction}

The increasing worldwide incidence of myopia corresponds with the increased prevalence of high myopia, especially in East Asia, where the prevalence of high myopia has been reported to range from 2.4 to $4.2 \%$ in the population over 30 years old. ${ }^{1}$ High myopia is also called pathological myopia, for its characteristic axial elongation, posterior staphyloma, and the consequential series of choroidoretinal lesions. The principle cause of vision loss in pathologic myopia is myopic maculopathy. Myopic maculopathy was first defined by Curtin and Karlin $^{2}$ in 1971, when the manifestation of degenerative changes such as chorioretinal atrophy, a central pigment spot ('Fuchs' spot'), lacquer cracks, posterior staphyloma, and optic disc changes were described. Since then, several photographic grading schemes have been proposed, but the definition of myopic maculopathy has not been consistent across studies. ${ }^{3}$ It was only when optical coherence tomography (OCT) was adopted that the study of the posterior vitreoretinal anatomy was attainable, which allowed the detection of subtle macular changes, such as retinoschisis, lamellar holes, or shallow macular detachment, and the important role of retinal traction generated by the epiretinal membrane (ERM) and/or the vitreomacular traction (VMT) were emphasized in the generation of myopic maculopathy. In 2004, Panozzo unified all of the pathologic features generated by traction in the myopic environment under the name of myopic traction maculopathy (MTM). ${ }^{4}$ According to Panozzo's classification, ${ }^{4}$ MTM is partially related to epiretinal traction forces and is then enhanced by the presence of staphyloma. The MTM can either present in isolation or in combination with 
other degenerative non-MTM myopic pathologic changes, such as choroid atrophy, lacquer cracks, retinal thinning, choroidal neovascular membranes, spontaneous foveal hemorrhage, or macular holes with or without posterior retinal detachment.

Although myopic maculopathy can be classified into MTM and non-MTM changes, the natural history of MTM and the differences between the two are not fully understood. Studies have shown that almost half of high myopic eyes have macular epiretinal traction, which was much higher than in eyes without myopia. This indicates that the scleral stretching of globe enlargement and staphyloma may generate an external force that greatly enhances the internal traction from epiretinal and vitreomacular adhesions. Thus, the comparison of the differences in morphological change between MTM and non-MTM eyes, along with a quantitative measurement of the globe, may reveal the pathological process and potential causes of MTM. Recently, Yoshimura ${ }^{5}$ evaluated the posterior pole curvature of eyes with MTM by OCT and demonstrated that sharp-pointed shape changes in the foveal area seemed to promote MTM in highly myopic eyes. However, the OCT scan was limited to the macular area, and the exact shape of the entire posterior segment of the eye was not obtained. In addition, the deep excavation of the posterior segment of highly myopic eyes prevents acquiring distinct OCT images. Thus a more fullscale and comprehensive understanding of the relationship between eye shape and MTM change still needs to be worked out.

Three-dimensional magnetic resonance imaging (3D MRI) has recently been reported to be well suited for the examination of eye shape over a wide area that can encompass even a large posterior staphyloma. ${ }^{6}$ This technique also allows the technician to view a staphyloma from any angle. Based on the study of the 3D MRI image of the eyeball, Moriyama ${ }^{7}$ proposed a simple classification of staphyloma on the grounds of Spaide's definition ${ }^{8}$ of a staphyloma as 'an outpouching of the wall of the eye that has a radius of curvature that is less than the surrounding curvature of the wall of the eye', instead of Curtin's classification, ${ }^{9}$ which was based on ophthalmoscopic appearances and fundus drawings. According to Moriyama's classification, ${ }^{7}$ the definition of staphyloma is when 'a second curvature develops in the posterior portion of the eye, and this second curvature has a shorter radius of curvature than the surrounding eye wall' and 'nasally distorted type of globe', which we here simplified to a 'non-uniform expansion' of the globe. In contrast, the 'uniform expansion' of the globe was defined as 'axial length elongation occurring in the equatorial region that does not induce any alteration in the curvature of the posterior part of the eye'.
In conclusion, the aim of this work was to study the characteristics of globe shape by 3D MRI in eyes with myopic maculopathy, and to quantitatively evaluate the differences between MTM and nonMTM eyes.

\section{Materials and methods \\ Study participants and groups}

All patients with high myopia (defined by a refractive error of -6 diopters or less or an axial length of $26.5 \mathrm{~mm}$ or more) in whom myopic maculopathy was confirmed by OCT were recruited in the Retina Department of Zhongshan Ophthalmology at Sun Yat-sen University from September 2015 to September 2016. We excluded eyes that had undergone vitrectomy, scleral buckling surgery, retinal laser photocoagulation, or anti-vascular endothelial growth factor treatment. All participants underwent comprehensive ocular examinations, including refractive error (spherical equivalent) measurements, axial length measurement, OCT scan, and 3D MRI scan. All procedures conformed to the tenets of the Declaration of Helsinki, and their use was approved by the Ethics Committee of the Zhongshan Ophthalmic Center, Sun Yat-sen University. All patients were fully informed of the study purpose and procedures, and written consent was obtained from each patient.

All of the myopic macular alterations were classified into two groups according to the definition of MTM (the MTM group) or else were included in the non-MTM group, as analyzed by spectral-domain optical coherence tomography (SD-OCT) (Carl Zeiss). According to Panozzo et al, the pathologic features generated by traction induced by the ERM and/or residual focal vitreoretinal adhesion VMT in the myopic environment were defined as MTM, which mainly included: (1) Retinal thickening: foveal thickness $>200 \mu \mathrm{m}$ with or without cystoid macular changes; (2) Macular retinoschisis: separation of the neurosensory retina into two or more layers; (3) Retinal detachment: shallow neuroepithelial detachment; or (4) Lamellar macular hole. Any of these types of damage could be present in isolation or in combination, in order to qualify as MTM. In addition, the cases of myopic maculopathy without MTM mostly consisted of degenerative changes, such as progressive atrophy of the choroid, lacquer cracks, retinal thinning, and the resulting lesions like choroidal neovascular membranes, spontaneous foveal hemorrhage, and macular holes with or without posterior retinal detachment. 


\section{D MRI analysis}

All patients were examined with a whole-body MRI scanner (Magnetom Verio 3.0T; Siemens, AG Healthcare, Erlangen, Germany) with a 12-channel phased-array head coil. The patients were told to close their eyes and keep them still during the scan. The MRI sequence was fatsuppressed T2-weighted SPACE (Sampling Perfection with Application optimized Contrast using different angle Evolutions, a 3D TSE variant) with the parameters of repetition time set to $1000 \mathrm{~ms}$, echo time to $200 \mathrm{~ms}$, echo train length to 75 , field of view to $200 \times 200$, matrix set as $384 \times 384$, and slice thickness as $0.5 \mathrm{~mm}$. This sequence showed a good isotropic spatial resolution of $0.5 \mathrm{~mm}$. The scanning time was 06:35. Because T2weighed images were used, the outer surface of the eye in 3D MRI shows the outer surface of the intraocular fluid. Thus, it depicts the inner surface of the retina in the posterior segment of the 3D MRI. The 3D MRI analyses of the globe, including volume renderings of the images, were compiled from high resolution 3D data on a VITAL 6.7.1 computer workstation (Toshiba Medical Systems Group Company). The margin of the globe was identified semiautomatically by the signal intensity, and the tissues on the outside of the globe were carefully removed.

According to the shape of the posterior pole of the globe, we classified either uniform or non-uniform expansion.

Quantitative evaluation of the globe included the vitreous volume, anteroposterior diameter (axial length, AL), equatorial diameter (ED), and the ratio of anteroposterior/equatorial diameter (AL/ED) (Figure 1). All of the image processing and analysis was completed by two specialized technicians who were masked to the maculopathy status of the patients.

\section{Statistical analysis}

The differences in the patient ages, refractive errors (spherical equivalent), vitreous volume, $\mathrm{AL}, \mathrm{ED}$, and AL/
ED between the two groups (MTM vs non-MTM) were analyzed with a two-tailed $t$-test (conformed to a normal distribution) or Mann-Whitney $U$-tests (did not conform to a normal distribution). The distributions of the shape of the globe in the two groups were analyzed by $\chi^{2}$-tests. A $P$-value $<0.05$ was considered statistically significant.

\section{Results}

A total of 105 eyes from 66 consecutive patients with high myopic maculopathy were recruited. Among them, two eyes from one patient were excluded because of a lowquality MRI, which was mainly owing to eye movements during the MRI examination. In the end, 103 eyes from 65 patients ( 24 males and 41 females) were studied. The mean age of the 65 patients was $54.57 \pm 12.71$ years with a range of 30-79 years. The mean refractive error (excluding pseudophakic, aphakic, or post-refractive surgery eyes) of the 103 eyes was $-12.70 \pm 5.37 \mathrm{D}$ with a spherical equivalent range from $-6.125 \mathrm{D}$ to $-31.250 \mathrm{D}$. The mean axial length was $28.95 \pm 2.20 \mathrm{~mm}$ with a range of $23.05-$ $34.15 \mathrm{~mm}$.

According to the OCT appearance and Panozzo's classification, 68 and 35 eyes were classified into the MTM and non-MTM groups, respectively. A comparison of the two groups is shown in Table 1 . There was no significant difference in age between the two groups $(P>0.05)$. The refractive error (spherical equivalent) of the non-MTM group was significantly more myopic than that of the MTM group $(P<0.05)$. Other quantitative evaluations showed that there was no significant difference found in either vitreous volume or other diameter parameters (including axial length, equatorial diameter, and axial length/equatorial diameter ratio) between the two groups $(P>0.05)$. However, we found that the distributions of the globe shape between the two groups showed a significant difference $(P<0.01)$. A less-uniform shape was found in the MTM group, whereas the non-MTM group tended to present eyes with a more uniform shape (Table 2).

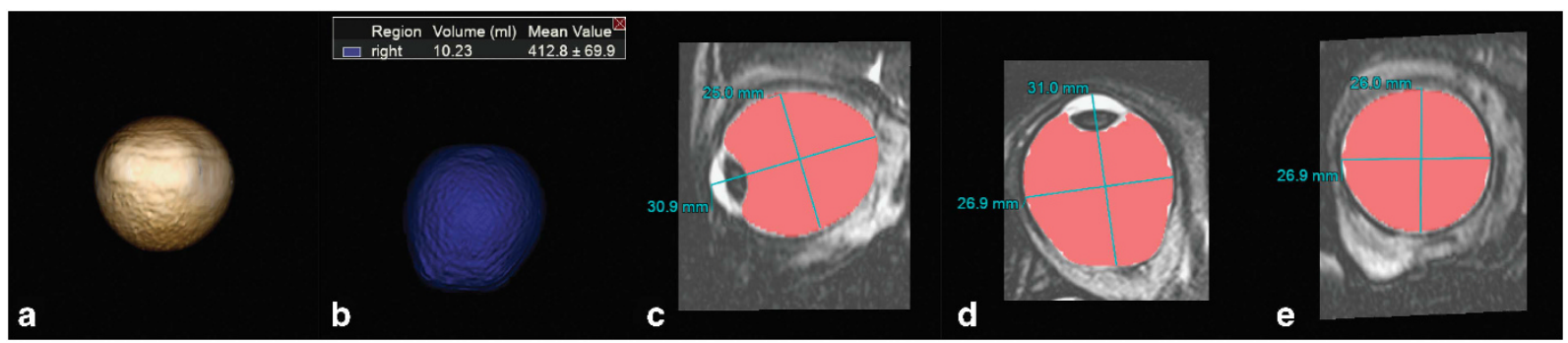

Figure 1 3D MRI volume renderings of the eye and quantitative analysis of the vitreous volume, anteroposterior diameter, axial length (AL) and equatorial diameter (ED). Volume renderings of three-dimensional magnetic resonance imaging (3D MRI) of the eyeball were performed. (a) the 3D images of the posterior pole of the eyeball (view from the back); (b) the vitreous volume was automatically calculated; (c-e) the calculation of three-dimensional diameters in three cardinal axes. Notice that each diameter was calculated twice and the average value was used. 
Table 1 Quantitative evaluation of the globe between the MTM group and the non-MTM group

\begin{tabular}{lccc}
\hline & MTM group & Non-MTM group & P-value \\
\hline Eye & 35 & 68 & 55 \\
Age (y/o) & 54 & $-16.52 \pm 1.16$ & 0.60 \\
Refractive error (spherical equivalent, diopter) & $-13.30 \pm 0.6321$ & 7.82 & $0.03^{\mathrm{a}}$ \\
Vitreous volume (ml) & 7.13 & 29.80 & 0.13 \\
Axial length (mm) & 28.80 & $24.11 \pm 0.20$ & 0.21 \\
Equatorial diameter (mm) & $24.09 \pm 0.11$ & 5.35 & 0.93 \\
AL/ED & 4.65 & 0.28 \\
\hline
\end{tabular}

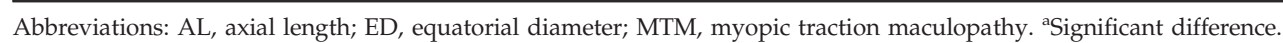

Table 2 Distributions of globe shape in the MTM and the non-MTM groups

\begin{tabular}{lcccc}
\hline & Non-uniform expansion & Uniform expansion & Total & P-value \\
\hline MTM group (eye) & 49 & 19 & 68 & $<0.0001^{\text {a }}$ \\
Non-MTM group (eye) & 7 & 28 & 35 & 103 \\
Total (eye) & 56 & 48 & \\
\hline
\end{tabular}

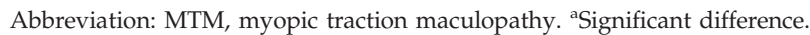

\section{Discussion}

In MTM, the posterior retina lies in an unstable condition determined by two opposite forces: the internal traction generated by the ERM or VMT, and the external traction of progressive scleral stretching and staphyloma. There was a higher frequency of epiretinal traction in pathologic myopic eyes than in eyes without myopia, indicating that the external traction, although not an active form of traction, greatly enhanced the internal traction in myopic eyes. Early studies have also inferred that the eye/retinal shape is associated with the onset of myopic maculopathy. ${ }^{9-11}$ Nevertheless, the classification of myopic maculopathy varies among studies and few specifically analyzed the eye shape in MTM. In addition, most of the studies analyzed the eye shape by traditional B ultrasonography or OCT scans, which was relatively limited in topography and quantitative analysis. Our study was the first to use the 3D MRI technique to characteristically analyze the eye shape in eyes with MTM.

Our study found a significant difference in eye shape distribution between MTM and non-MTM eyes. Most of the MTM eyes had a non-uniform expansion of the eyeball, whereas the non-MTM eyes mostly had a uniform expansion. No significant differences were found, however, in eyeball diameter (regardless of axial length, equatorial diameter, or even the axial length/ equatorial diameter ratio) between MTM and non-MTM eyes. In addition, the comparison of vitreous volume showed no significant difference between the two groups. The partial eyeball bulge and staphyloma formation might be closely related to MTM pathogenesis, rather than general eyeball enlargement.
The importance of staphyloma has been noted by previous studies. Hsiang ${ }^{12}$ demonstrated that the incidence of macular disease in myopic eyes increased with increasing staphyloma grade. Rahimy ${ }^{13}$ used SDOCT to confirm that increasing staphyloma curvature was significantly associated with a higher incidence of myopic maculopathy. Our study indicates that nonuniform globe expansion and staphyloma formation might have an important role in the pathogenesis of MTM. In consideration of the mechanism of traction maculopathy, we might infer that the uneven scleral stretching, particularly that at the edge of the staphyloma, could increase the radian of eyeball and thereby enhance the relative force between epiretinal/vitreomacular adhesions and the scleral wall, which would generate a large amount of tangential shear stress at the inner surface of the retina and finally lead to the traction lesion in MTM eyes. Our results also revealed that the degree of axial elongation or general globe expansion may be less decisive in MTM formation than was previously believed. Curtin ${ }^{14}$ reported that the axial length might not be a good indicator for the characterization of pathologic myopia when the eye is deformed and not spherical. For this reason, we calculated the three-dimensional eyeball diameters, as well as their ratios. However, the MTM and non-MTM eyes showed no difference for any of the cardinal axes. More importantly, the comparison of vitreous volume also supported the above results. Previous studies have proven the enlargement of myopic eyes compared with emmetropic eyes by MRI, and that the increase in eye volume was mostly determined by the increase in vitreous volume. ${ }^{15,16}$ Thus, the indifferent vitreous volume comparison further proved the consistent degree of general globe expansion of MTM and 
non-MTM eyes, which demonstrates that general globe expansion is not a determined factor in the formation of MTM. In addition, we were unable to explain the results that the non-MTM eyes were significantly more myopic than those from the MTM group, which will require further study.

The surgical management of MTM is still debated. The elimination of epiretinal tractions by means of vitrectomy with or without internal limiting membrane peeling has proven to be effective. ${ }^{17,18}$ Nevertheless, this method has the risk of complications, such as macular hole formation or extrafoveal retinal hole formation, and is still associated with a high retinal detachment recurrence rate. ${ }^{19,20}$ The use of a scleral buckle to reduce the tractions secondary to staphyloma has been reported with good anatomical and functional results. ${ }^{21-24}$ Recent studies have shown that macular buckles are safe and well tolerated, even after long-term follow-up, and have a very low rate of complications. Therefore, the authors suggested that macular buckles should be used alone as the first intervention, whereas vitrectomy should be used in combination with a macular buckle or as a second-line approach in MTM eyes. ${ }^{25,26}$ The results of our study also support the view that the macular buckle was indispensable, not to shorten the axial length, but to fulfill a more important role by flattening the posterior staphyloma, in order to prevent the long-term complications linked to the progressive worsening of the staphyloma.

There were several limitations of our study. First, the sample size was relatively small, especially the non-MTM group, and because patients with more severe symptoms tend to agree more readily to 3D MRI examination, more extremely myopic patients were enrolled. Second, the nature of the cross-sectional study limited the observation of the natural disease course and prevented further research into the correlation of eye shape change and the development of maculopathy. Last but not least, although the quantitative evaluation was achieved in the calculation of diameter, the morphological analysis of the posterior pole and staphyloma was qualitative and relatively subjective. We had all the image processing and analysis finished by two specialized technicians with agreeable classification standards, and divergence was proven to be rare and was carefully analyzed and excluded.

In conclusion, our study was the first to use the 3D MRI technique to characteristically analyze the eye shape of MTM. Non-uniform globe expansion and staphyloma formation were closely associated with MTM. This information should be useful to further the understanding of the pathogenesis and natural history of different types of myopic maculopathy, which will ultimately promote the prevention and treatment of myopic maculopathy.

\section{Summary}

\section{What was known before}

- The increasing incidence rates of myopia worldwide have also increased the prevalence of high myopia, especially in East Asia, where the prevalence of high myopia has been reported to range from 2.4 to $4.2 \%$ in the population over $30-40$ years old.

What this study adds

- The ununiformed globe expansion and staphyloma formation was a determined factor in the onset of MTM.

\section{Conflict of interest}

The authors declare no conflict of interest.

\section{Acknowledgements}

This work was supported by the General Program (81570862) of the National Natural Science Foundation of China (to LL) and the General Program (81371019) of the National Natural Science Foundation of China (to YL). The funding organizations had no role in the design or conduct of this research.

\section{References}

1 Verkicharla PK, Ohno-Matsui K, Saw SM. Current and predicted demographics of high myopia and an update of its associated pathological changes. Ophthalmic Physiol Opt 2015; 35(5): 465-475.

2 Curtin BJ, Karlin DB. Axial length measurements and fundus changes of the myopic eye. Am J Ophthalmol 1971; 71(1): $42-53$.

3 Ohno-Matsui K, Kawasaki R, Jonas JB, Cheung CM, Saw SM, Verhoeven VJ et al. International photographic classification and grading system for myopic maculopathy. Am J Ophthalmol 2015; 159(5): 877-83 e7.

4 Panozzo G, Mercanti A. Optical coherence tomography findings in myopic traction maculopathy. Arch Ophthalmol 2004; 122(10): 1455-1460.

5 Wakazono T, Yamashiro K, Miyake M, Nakanishi H, Oishi A, Ooto $\mathrm{S}$ et al. Association between eye shape and myopic traction maculopathy in high myopia. Ophthalmology 2016; 123(4): 919-921.

6 Moriyama M, Ohno-Matsui K, Hayashi K, Shimada N, Yoshida T, Tokoro T et al. Topographic analyses of shape of eyes with pathologic myopia by high-resolution threedimensional magnetic resonance imaging. Ophthalmology 2011; 118(8): 1626-1637.

7 Moriyama M, Ohno-Matsui K, Modegi T, Kondo J, Takahashi Y, Tomita M et al. Quantitative analyses of high-resolution 3D MR images of highly myopic eyes to determine their shapes. Invest Ophthalmol Vis Sci 2012; 53(8): 4510-4518.

8 Spaide RF. Staphyloma: Part 1. In: Spaide RF, Ohno-Matsui K, Yannuzzi LA, eds. Pathologic Myopia. New York: Springer; 2013: 167-176. 
9 Curtin BJ. The posterior staphyloma of pathologic myopia. Trans Am Ophthalmol Soc 1977; 75: 67-86.

10 Pruett RC. Complications associated with posterior staphyloma. Curr Opini Ophthalmol 1998; 9(3): 16-22.

11 Steidl SM, Pruett RC. Macular complications associated with posterior staphyloma. Am J Ophthalmol 1997; 123(2): 181-187.

12 Hsiang HW, Ohno-Matsui K, Shimada N, Hayashi K, Moriyama M, Yoshida T et al. Clinical characteristics of posterior staphyloma in eyes with pathologic myopia. Am J Ophthalmol 2008; 146(1): 102-110.

13 Rahimy E, Beardsley RM, Gomez J, Hung C, Sarraf D. Grading of posterior staphyloma with spectral-domain optical coherence tomography and correlation with macular disease. Can J Ophthalmol 2013; 48(6): 539-545.

14 BJ C. Ocular Findings and Complications. The Myopias: Basic Science and Clinical Management. Harper and Row: Philadelphia, PA, USA: 1985; pp 297-299.

15 Cheng HM, Singh OS, Kwong KK, Xiong J, Woods BT, Brady TJ. Shape of the myopic eye as seen with highresolution magnetic resonance imaging. Optom Vis Sci 1992; 69(9): 698-701.

16 Verkicharla PK, Mathur A, Mallen EA, Pope JM, Atchison DA. Eye shape and retinal shape, and their relation to peripheral refraction. Ophthalmic Physiol Opt 2012; 32(3): 184-199.

17 Gonvers M, Machemer R. A new approach to treating retinal detachment with macular hole. Am J Ophthalmol 1982; 94(4): 468-472.

18 Kanda S, Uemura A, Sakamoto Y, Kita H. Vitrectomy with internal limiting membrane peeling for macular retinoschisis and retinal detachment without macular hole in highly myopic eyes. Am J Ophthalmol 2003; 136(1): 177-180.
19 Ikuno Y, Sayanagi K, Ohji M, Kamei M, Gomi F, Harino S et al. Vitrectomy and internal limiting membrane peeling for myopic foveoschisis. Am J Ophthalmol 2004; 137(4): 719-724.

20 Panozzo G, Mercanti A. Vitrectomy for myopic traction maculopathy. Arch Ophthalmol 2007; 125(6): 767-772.

21 Ripandelli G, Coppe AM, Fedeli R, Parisi V, D'Amico DJ, Stirpe M. Evaluation of primary surgical procedures for retinal detachment with macular hole in highly myopic eyes: a comparison [corrected] of vitrectomy versus posterior episcleral buckling surgery. Ophthalmology 2001; 108(12): 2258-2264.

22 Theodossiadis GP, Sasoh M. Macular buckling for retinal detachment due to macular hole in highly myopic eyes with posterior staphyloma. Retina 2002; 22(1): 129.

23 Theodossiadis GP, Theodossiadis PG. The macular buckling procedure in the treatment of retinal detachment in highly myopic eyes with macular hole and posterior staphyloma: mean follow-up of 15 years. Retina 2005; 25(3): 285-289.

24 Liu B, Ma W, Li Y, Luo Y, Jin C, Liang X et al. Macular buckling using a three-armed silicone capsule for foveoschisis associated with high myopia. Retina 2016; 36 (10): 1919-1926.

25 Parolini B, Frisina R, Pinackatt S, Mete M. A new L-shaped design of macular buckle to support a posterior staphyloma in high myopia. Retina 2013; 33(7): 1466-1470.

26 Parolini B, Frisina R, Pinackatt S, Gasparotti R, Gatti E, Baldi A et al. Indications and results of a new l-shaped macular buckle to support a posterior staphyloma in high myopia. Retina 2015; 35(12): 2469-2482. 